\title{
Hydrogen release and retention from porous graphite
}

\author{
A. Rai ${ }^{1}$, R. Schneider ${ }^{1}$ and M. Warrier ${ }^{2}$ \\ ${ }^{1}$ Max-Planck-Institut für Plasmaphysik, D-17491 Greifswald, Germany \\ ${ }^{2}$ Beam Technology Development Group, BARC, Trombay, Mumbai, India - 400085
}

\begin{abstract}
The retention and release of implanted hydrogen atoms and molecules from 3-D porous graphite was studied. Kinetic Monte-Carlo calculations were used to study reactive-diffusive transport of hydrogen. We have analysed various factors affecting retention and release of hydrogen from graphite. It is shown that the internal structure of the graphite (void fraction, void size and void orientation), the energy of the incident hydrogen ion beam and the flux plays a major role in retention and release behaviour.
\end{abstract}

PACS: 02.70.Uu

Keywords : Diffusion

Corresponding author: Abha Rai

Phone: +49 3834 882400, E-mail: Abha.Rai@ipp.mpg.de 


\section{Introduction}

The understanding of retention and release of hydrogen in graphites and carbon-based materials is important for fusion plasma density control and for the determination of tritium inventory in future fusion machines. The purpose of this research study is to understand the transport of hydrogen and its interaction with graphite. Various factors which affect the retention and release process of hydrogen in graphite are analysed. The graphites used as plasma facing material (PFM) in fusion devices are porous and consist of granules (few microns in size) separated by voids (fraction of a micron in size). These granules further consist of randomly oriented crystallites (a few nanometer in size) separated by micro-voids (fraction of nanometer in size) [1,2].

We are interested in the analysis of thermalized hydrogen. The collisional cascade effects are taken into account by using for the depth distribution corresponding to the range of the incident ions as calculated with TRIM. The ion beam energies we have considered in our simulation spans from 1-3 keV. We approximate the profile of the ions given by TRIM calculations to a Gaussian distribution with a range of penetration of 27.5 to $59.0 \mathrm{~nm}$ and a standard deviation of 8.0 to $12.0 \mathrm{~nm}$ along the $\mathrm{z}$-direction. The full width at half maximum of the distribution is 16.0 to $24.0 \mathrm{~nm}$. The basic cell size was $0.5 \mathrm{~nm}$. This implies that an ion beam having an energy of $1 \mathrm{keV}$ was centered

around the $55^{\text {th }}$ cell from the surface covering around 16 cells on both sides. Due to the short mean free path of hydrogen atoms (fraction of a nanometer) it is sufficient to concentrate on the transport mechanisms in the trans-granular level (within one 
granule with dimensions about $100 \mathrm{~nm}$ or 200 cells in $\mathrm{z}$-direction) for retention and release studies. A typical granule consists of a bulk region within the crystallites, connected by intercrystalline passageways (inner surface region) [3]. Hydrogen ions and neutrals which are not reflected have a wide range of possibilities as discussed in $[2,1,4,5]$. Deposited hydrogen atoms will first diffuse within the crystallites until it reaches the crystallite microvoid interface where it can be trapped or it reaches the crystallite surface. A hydrogen atom then diffuses along the microvoid-crystallite interface until it recombines (if possible) or until it reaches the real surface, from where it can be released with a temperature-dependent probability. Diffusion of hydrogen atoms along the crystallite surface leads to the possibility of hydrogen atom penetration well beyond the implantation zone. Transport of hydrogen atoms from the crystallite surface back into the crystallites is limited by a potential energy barrier. Molecule formation happens when two hydrogen atoms diffuse and come closer than a certain distance of the order of a few angstroms. Therefore unless there is a high density of hydrogen atoms, molecule formation does not happen at the end of the range.

Chiu and Haasz [6] found that there exist two different diffusion channels in the crystallites, with different migration energies and prefactors. They speculated that this was due to the diffusion of hydrogen atoms along graphite planes within the crystallites at low temperatures $(<500 \mathrm{~K})$, whereas at higher temperatures $(>500 \mathrm{~K})$ diffusion (perhaps through defects) could occur perpendicular to the planes. They observed a difference in the release of HD molecules when a pseudo-monocrystal graphite, preimplanted with $1 \mathrm{keV} \mathrm{D}^{+}$, was bombarded with $10 \mathrm{keV} \mathrm{H}^{+}$perpendicular or parallel to 
the graphite planes indicated an inhibited diffusion across the planes, but a free diffusion along the planes at room temperature. The MD studies by Warrier [7] reveales that two channels do exist but there is no diffusion across the graphene layers and the diffusion is isotropic in the plane of the graphene layers. Phonon vibrations of the graphene layers show a large amplitude at higher temperatures $(>450 \mathrm{~K})$. This allows the hydrogen interstitials to sneak through the large openings between the graphene layers and by interaction with the phonons, allowing longer jumps.

The existence of such large variations in length scales of sub-structures coupled with the wide range of possible atomistic processes makes the study of hydrogen transport and inventory (or complementing this, the formation of hydrocarbons and their transport) in graphite a non-trivial exercise. Many detailed macroscopic models have been proposed to study hydrogen inventory and transport in porous graphite $[2,1,4,8]$ and hydrocarbon formation and transport in graphite [9]. These models use rate constants for transport from experiments [10-14,5], some of which still need theoretical explanations. There exist many microscopic models [15-18] using MD with either empirical potentials or density functional theory and they give insight into the microscopic mechanisms studied in graphite. It is desirable to use the insights gained from the microscopic models for modeling the transport in the meso-scale and further into the macro-scale in order to understand the physical processes contributing to macroscopic transport.

We have earlier modeled hydrogen diffusion in crystal graphite using MD at microscales $\left(2.5 \mathrm{~nm}, 10^{-10} \mathrm{~s}\right)$ and consistently parametrized the MD results within a Kinetic 
Monte-Carlo (KMC) scheme [18]. A 3D porous granular structure was constructed using statistical distributions for crystallite dimensions and crystallite orientations for a specified micro-void fraction. The KMC scheme was extended to include trapping and detrapping at the crystallite-microvoid interface in the $3 \mathrm{D}$ porous granule structure to simulate trans-granular-diffusion (TGD) in the mesoscales $\left(10^{-7}-10^{-6} \mathrm{~m}\right.$, several $m s)$ [19] using the results from our microscale modeling and from experiments ([20] and references therein). Later, this concept was extended in our simulations to the macro-scales $(1 \mathrm{~cm}$, up to a few $s)$, thereby having a truly multi-scale capability [21]. The simulations at the meso-scales were only for trace amounts of hydrogen atoms which had a very low probability of forming molecules.

In this paper, we use an extension of this model. We introduce molecular species in addition to the atomic species to study the hydrogen transport and retention in and release from a 3-D porous graphite structure. The code was modified by using the Smoluchowski boundary condition [22] to model specie recombination. This is achieved by checking the distance of hydrogen atoms. If this distance gets smaller than a certain distance $\mathrm{r}_{o}$ of each other they recombine to form hydrogen molecules. This is equivalent to having a step potential well for interaction of the species at a radial distance $\mathrm{r}_{o}$. Such boundary condition is valid for the cases with strong binding energies (like hydrogen recombination or hydrogen trapped at the open bond site) where reflection becomes a rare event once the atom gets closer than a cutoff distance [23]. Apart from diffusion and detrapping, we have included other reactions e.g. recombination, trapping, dissociation. This changes the characteristics of the code from a "trace atom 
diffusion" code to a "reactive-diffusive" code. Within the KMC ansatz, all attractive potentials are considered as traps, be it either covalent bonding or adsorption. Therefore, we have loosely used the term trap for even adsorption events. We also refer to the energy for an atom to (i) detrap, (ii) dissociate or (iii) desorb as the migration energy. This is because any of these events leads to a migration of the trapped atom. The model incorporates experimental findings on hydrogen molecule formation and atomic hydrogen diffusion in graphites. Model calculations will be compared with experimental results on retention and release of hydrogen from graphite.

\section{Model}

The parametrization of the processes for the molecules is based on [1]. A two-region model is implemented distinguishing for atoms or molecule transport processes within the bulk and surface region on the graphite crystallites. Molecules in the crystallites can undergo a simple jump, characterized in terms of the KMC parameters migration energy $(2.0 \mathrm{eV})$, jump length $(0.3 \mathrm{~nm})$ and jump attempt frequency $\left(2.74 \cdot 10^{13} \mathrm{~s}^{-1}\right.$ (typical phonon frequency)). This energy is high compared to the energy needed by hydrogen molecules to jump in the voids. This assumption is justified by the small interlayer spacing within the graphite crystallites, which makes the jumps for hydrogen molecules difficult due to their size. They can also experience a dissociation $\left(4.45 \mathrm{eV}, 0.2 \mathrm{~nm}, 2.74 \cdot 10^{13} \mathrm{~s}^{-1}\right)$ [24]. Within the voids we consider simple jumps

or desorption $\left(0.06 \mathrm{eV}, 1.0 \mathrm{~nm}, 1.0 \cdot 10^{13} \mathrm{~s}^{-1}\right)$ or dissociation $(4.45 \mathrm{eV}, 0.2 \mathrm{~nm}, 1.0$. 
$10^{13} s^{-1}$ ). The radius for the recombination of hydrogen atoms is in the range 0.17 $0.27 \mathrm{~nm}$ [25], we use a value of $0.2 \mathrm{~nm}$. Atoms in the crystallites can have either short $\left(0.015 \mathrm{eV}, 0.38 \mathrm{~nm}, 6.8 \cdot 10^{12} \mathrm{~s}^{-1}\right)$ or long $\left(0.269 \mathrm{eV}, 0.1 \mathrm{~nm}, 2.74 \cdot 10^{13} \mathrm{~s}^{-1}\right)$ jumps. Atoms on the crystallite-microvoid interface (CMI) can jump along the CMI (0.09 eV, $\left.0.34 \mathrm{~nm}, 1.0 \cdot 10^{13} \mathrm{~s}^{-1}\right)$, can enter the crystallites $\left(2.67 \mathrm{eV}, 0.4 \mathrm{~nm}, 1.0 \cdot 10^{13} \mathrm{~s}^{-1}\right)$ or can be desorbed $\left(1.9 \mathrm{eV}, 0.2 \mathrm{~nm}, 1.0 \cdot 10^{13} \mathrm{~s}^{-1}\right)$ from the surface. A trapped hydrogen atom can get detrapped $\left(2.67 \mathrm{eV}, 0.3 \mathrm{~nm}, 1.0 \cdot 10^{13} \mathrm{~s}^{-1}\right)$ and become a mobile hydrogen atom. The jump "entering into the crystallite" corresponds to the jump when a hydrogen atom is trapped at the edges of the crystallite. When a detrapping event takes place, the resulting mobile hydrogen atom can either jump into the crystallite or into the void. It also incorporates the potential well as seen by a mobile hydrogen coming close to a crystallite edge. There is also a possibility that a mobile hydrogen atom recombines with a trapped hydrogen atom at the crystallite-microvoid interface to form a hydrogen molecule. MD studies are needed to do the parametrization for the release of such hydrogen molecules. In the present model we have not included this process.

In the present work, the number of hydrogen atoms and trap sites are specified according to a Gaussian profile based on the energy of the ion beam we want to simulate and then follow the evolution of the system. We calculate the amount of hydrogen released and retained in the sample. From the released amount of hydrogen we calculate the fraction of hydrogen released in the form of atoms and molecules. There is no continuous influx of the hydrogen ions, therefore with the present model we can not 
simulate the dynamic buildup of hydrogen inventory and re-emission after the steady state is achieved. The main purpose of this work is to identify different physical processes taking place in the system and different factors influencing them. Since there is no continuous influx of hydrogen ions it is not possible to define a flux or fluence value for hydrogen ions in this work. For comparison of the results and the trends observed in the simulations we associate the term fluence to the atomic density of hydrogen i.e. the number of hydrogen atoms present per unit area. All the simulations are done for same duration $\left(\simeq 10^{-7} s\right)$, therefore an increase in fluence corresponds to the increase in the initial number of hydrogen atoms introduced in the sample.

\subsection{Validation of the model}

For validating our model, we simulate an experiment used to study the plasma-surface interaction at KFA Jülich [26]. Graphite samples were bombarded by a $3 \mathrm{keV} H_{3}^{+}$ion beam at normal incidence. The ion beam had a fluence of $10^{18} \mathrm{H} / \mathrm{cm}^{2}$. Re-emitted particles were directly detected by a two-stage differentially pumped line-of-sight quadrupole mass spectrometer. Several data points were presented for each temperature in order to document the scatter in the data. The material chosen in the experiment was EK98 ( Ringsdorff, FRG), an isotropic, porous, fine grain graphite with the density $1.85 \mathrm{~g} / \mathrm{cm}^{3}$. This gives a rough estimate of the void fraction of about $12 \%$ (density of standard graphite is about $2.09-2.23 \mathrm{~g} / \mathrm{cm}^{3}$ ). As mentioned earlier, the internal structure of the graphite plays a major role in the retention and release behaviour, so we did a parameter scan over different geometries of the graphite and 
found that the following geometrical configuration gives the best agreement with the experiment. The typical ion beam energies we have considered in our simulation spans from 1-3 keV. This corresponds to a depth distribution with range of penetration of $27.5-59.0 \mathrm{~nm}$. The basic cell size was $0.5 \mathrm{~nm}$. The graphite sample chosen for this case was a cube of $100 \mathrm{~nm} \times 100 \mathrm{~nm} \times 100 \mathrm{~nm}$ having a void fraction $\simeq 10 \%$ and a void size $1 n m \times 10 n m \times 10 n m$ in $\mathrm{x}, \mathrm{y}$ and $\mathrm{z}$ directions, respectively. The geometrical surface lies at $z=0$. We distributed 2000 hydrogen atoms with Gaussian profile at the depth of $27.5 \mathrm{~nm}$ and width of $15.6 \mathrm{~nm}$ as derived from TRIM calculations and follow the evolution of the system for identifying the different reactions taking place in the sample and their effect on retention and release of hydrogen. All the simulations were done for the duration of $1.0 \times 10^{-7} \mathrm{~s}$. This corresponds to simulating the effect of an ion beam having energy of $1 \mathrm{keV}$ and fluence of $10^{13} \mathrm{H} / \mathrm{cm}^{2}$. Next step to the improvement of the present code which includes the dynamic effect of the incoming ion-beam with a continuous influx of the hydrogen atoms in a true steady state is under progress and will be published soon.

Fig. 1 shows the released amount of hydrogen as a function of temperature. From the total released amount the fraction contributed by atoms and molecules is plotted on the Y-axis. Out of 2000 hydrogen atoms distributed initially in the sample around $10 \%$ hydrogen is relesed at $900 \mathrm{~K}$, increasing to $70 \%$ at $1500 \mathrm{~K}$ and finally saturating around $87 \%$ at $2100 \mathrm{~K}$. The released amount of hydrogen at different temperatures can be found from the corresponding retention curve (Fig. 2). The hydrogen release curve follows the experimental results of [26] and the model calculation of [1], i.e. the 
released flux is mainly molecular hydrogen at lower temperatures and atomic hydrogen at higher temperatures. At lower temperatures $(<700 \mathrm{~K})$, the desorption energy of the hydrogen atoms is too high $(1.9 \mathrm{eV})$ for $\mathrm{H}$ atom release. However it diffuses on the surface (migration energy $0.9 \mathrm{eV}$ ) and recombines to form hydrogen molecules. These molecules have a very low desorption energy $(0.06 \mathrm{eV})$ and almost immediately diffuse to the surface and are thermally released. As the temperature increases, $\mathrm{H}$ atomic desorption from the internal surfaces also becomes an important process and we begin to observe atomic hydrogen release too. Since atomic hydrogen release occurs, there are lesser hydrogen atoms for molecule formation and this results in a fall of molecular hydrogen release at higher temperatures. The released flux depends on the competition between diffusion, recombination and trapping-detrapping which further depends on the internal structure of the graphite. Graphite samples having different internal structure offer different amounts of inner surface area, which determines the amount of passage offered to hydrogen atom for diffusion towards the real surface or deep into the bulk and thus affect release and retention behaviour, respectively. The inner surface area of the sample also determines the recombination and trapping probability. The higher the inner surface area offered by the sample, the higher is the probability for recombination or trapping.

The temperature at which both atomic and molecular hydrogen are released in equal $\operatorname{amount}\left(\mathrm{T}_{50 \%}\right)$ is shifted about $200 \mathrm{~K}$ towards lower temperatures in our results compared to experiment. This is due to the fact that the amount of released atoms or molecules depend on the incoming flux. This shift was explained earlier already by 
Haasz et al. [1] and is due to the different flux value in our calculation. The released flux is basically determined by the competition of the second-order process of recombination with the first-order process of atomic hydrogen diffusion and release. In our simulation it was observed that with increasing the initial number of hydrogen atoms in the system, $\mathrm{T}_{50 \%}$ shifts towards higher temperatures and considerable amount of about $20-30 \%$ of the released hydrogen is released in molecular form even at higher temperatures. This point is discussed later, when we present the analysis of the dependence of the initial number of hydrogen atoms on the release characteristics.

Other parameters like internal structure, trapsite density, energy of the incident ion beam, also affect retention and release of hydrogen from graphite and it is a difficult task to identify the effect of those. For this reason, we did different sensitivity studies.

The retention of hydrogen in graphite is not limited to the ion implantation range. Due to the diffusion of the implanted hydrogen along the internal porosity surfaces, hydrogen can penetrate deep inside the bulk well beyond the implantation zone. A graphite sample exposed to a hydrogen ion beam retained all of the incident ion and no saturation was observed till about $10^{17} \mathrm{H}^{+} / \mathrm{cm}^{2}[27]$. It was shown that the retained amount of hydrogen in graphite depends on the temperature of the sample, the fluence of the ion beam and the type of graphite used. Fig. 2 shows the retained amount of hydrogen as a function of temperature. Results from other references [28-32] are also presented for comparison. At lower temperatures, the retention is practically $100 \%$ and as the temperatures increases, hydrogen is released and the retained amount reduces to about 5\%. As discussed before, at lower temperatures, practically all the 
hydrogen present in the sample is in the form of trapped hydrogen and the probability of detrapping event is very low. Even if some detrapping event takes place the resulting solute hydrogen atom gets retrapped in its course of diffusion and therefore most of the hydrogen is retained in the sample and nothing is released. Whereas at higher temperatures the detrapping starts and the diffusion coefficient of hydrogen also increases. This high mobility of atoms leads to higher desorption and lower retention values. As observed by Sawicki et al. [28], the retention characteristics depend strongly on the incident fluence and follows a regular trend. At a given temperature, for low fluences, the concentration of atomic hydrogen on the inner surfaces is lower. Therefore the recombination probability decreases. This gives rise to a reduced molecular hydrogen formation and lower desorption i.e. higher retention levels. Also, the longer the duration of the bombardment (higher fluence) the more time is available for hydrogen to diffuse. Consequently, the probability of an outdiffusing hydrogen atom to be released or recombine is higher which leads to higher desorption i.e lower retention levels.

\section{Results}

Based on the model described above, we study the influence of different factors affecting hydrogen retention and release from graphite. The internal geometry plays a major role in retention and release, so we will first keep the internal structure the same in all the cases. The effect of void fraction and void size was studied [33], and we have also shown that even the orientation of the voids affects the hydrogen release 
[This is to be published]. For a systematic study, we take a sample with cubic voids, neglecting here the further dependence on the voids. In our previous work [33] a sample with $\simeq 5 \%$ void fraction gave the best fit with the experimets. Therefore in the present sensitivity studies void fraction was chosen to be $\simeq 5 \%$ in order to compare the results with the earlier published results and analyse the effect of the additional diffusion channel (diffusion along the inner porosity surface, in earlier work we considered diffusion only across the inner surface). The graphite sample chosen was a $100 \mathrm{~nm} \times 100 \mathrm{~nm} \times 100 \mathrm{~nm}$ cube with a void fraction $\simeq 5 \%$ and a mean void size $10 \mathrm{~nm} \times 10 \mathrm{~nm} \times 10 \mathrm{~nm}$ in $\mathrm{x}, \mathrm{y}$ and $\mathrm{z}$ directions, respectively. The geometrical surface lies at $z=0$. As we use a poisson distribution to create the geometry there are closed as well as open pores in the sample. Closed pores are the voids which are not directly connected to the surface, whereas open pores are directly connected to the surface. The ion beam scenario we studied had a fluence of $2 \times 10^{13} \mathrm{H} / \mathrm{cm}^{2}$ and an energy of $\simeq$ $1.7 \mathrm{keV}$. This determines the mean range of penetration of the ion beam of $37.5 \mathrm{~nm}$ and a standard deviation of $7.8 \mathrm{~nm}$ from TRIM.

\subsection{Effect of range of penetration of incident ion beam}

Fig. 3 shows the effect of the ranges of penetration on the hydrogen release behaviour. Ion beams with ranges of penetration of $27.7 \mathrm{~nm}($ energy $\simeq 1 \mathrm{keV}), 37.5 \mathrm{~nm}$ (energy $\simeq 1.7 \mathrm{keV})$ and $47.5 \mathrm{~nm}($ energy $\simeq 2.4 \mathrm{keV})$ were used. All the simulations were done with initially 2000 hydrogen atoms in the system. The retained amount for all these simulation cases are given in Fig. 4 and one can find for each temperature the exact 
amount of hydrogen released from the retained amount $((100$ - retained amount $\%) \times$ initial number of hydrogen /100). The results agree well with those of Haasz et al. [1]. It is seen that as the energy of the incident ion beam is increased (which also means an increase in range of penetration) a larger fraction of hydrogen is released in molecular form than in atomic form. The higher the energy of the incident ion beam, the deeper it can penetrate. This results in a higher probabilty for atoms to recombine on an inner surface before they reach the real surface due to increased diffusion paths, thereby increasing the possibility of molecule formation. Also, the temperature at which both atomic and molecular hydrogen are released in equal amount $\left(\mathrm{T}_{50 \%}\right)$ is lower for an ion beam with a low range of penetration and the release of hydrogen in atomic form starts at lower temperatures. The closer the hydrogen atoms are distributed with respect to the real surface of hydrogen the easier it is for them to diffuse out and to reach the real surface without being trapped and recombined. The hydrogen release characteristics are governed by the competition between trapping-detrapping, recombination and desorption.

Fig. 4 shows that the net level of retention is higher in the case of an ion beam with higher energy as the specimen temperature is increased. This is expected, because the deeper the ions penetrate until thermalization, the higher is the probability of being trapped at trapping sites availabe at the CMI before reaching the real surface for release leading to higher retention levels. This figure is consistent with Fig. 3 and confirms the kind of logic presented before. 


\subsection{Effect of initial number of hydrogen atoms}

Fig. 5 shows the effect of initial number of hydrogen atoms introduced in the system on hydrogen release behaviour. We studied cases with 2000, 5000 and 8000 hydrogen atoms distributed in the sample with a Gaussian distribution in $\mathrm{z}$-direction. The temperature at which both atomic and molecular hydrogen are released in equal amount $\left(\mathrm{T}_{50 \%}\right)$ shifts towards higher temperatures by increasing the initial number of hydrogen atoms and a large fraction of molecules are released even at higher temperatures. With increasing the initial number of hydrogen atoms, the density of trapped and mobile hydrogen atoms on the inner surfaces increases. This gives rise to higher recombination and trapping probabilities which reduces the probability of a diffusing hydrogen atom to be released in atomic form. Even at very high temperatures, detrapping takes place, however, due to the very high probability of being recombined or trapped, the hydrogen release in atomic form is less probable as compared to cases with lesser amount of initial hydroegn atoms where due to lower density of hydrogen atoms on inner surfaces the probability of being trapped or recombined is smaller. Retention curves follow the expected behaviour as shown in Fig. 2, i.e. at a given temperature samples subjected to a lower fluence have a higher level of retention. At a given temperature, for low fluence the density of trapped or mobile hydrogen atom at the inner surfaces is less and after a detrapping event takes place there is very high probability that the mobile hydrogen atom gets retrapped again at a trapping site. The probability of meeting another hydrogen atom and recombining to form a molecule is less due to the low due to the density of hydrogen atom. This leads to the high retention levels for lower 
fluences at a given temperature.

\subsection{Effect of trap site density}

Fig. 6 shows the effect of trap site density on the hydrogen release behaviour. Three runs were carried out with a trap density varying from 1.5 to $2.5 \times 10^{13}$ trap sites $/ \mathrm{cm}^{2}$. There are more trapped hydrogen atoms in case of the higher trap density but neither the retained nor the released amount of hydrogen is influenced. In our earlier work [33], we saw that there was a shift in $\mathrm{T}_{50 \%}$ (the temperature at which both atomic and molecular hydrogen are released in equal amount) towards lower temperatures for higher trap site density $\left(2.0\right.$ to $4.0 \times 10^{13}$ trap sites $\left./ \mathrm{cm}^{2}\right)$. This difference is attributed to the fact that in our earlier work we had diffusion of hydrogen mainly across the CMI (with migration energy $0.9 \mathrm{eV}$ ) which resulted in desorption. It was suggested by Haasz et al. [1] that a more realistic mechanism for the migration of a solute hydrogen atom on the inner surface is diffusion along the inner porosity, therefore, in the present work, we consider diffusion along the CMI ( with migration energy $0.9 \mathrm{eV}$ ), which naturally provides more access to the availabe traps and consequently more trapped hydrogen atoms and finally desorption (with migration energy $1.9 \mathrm{eV}$ ). Therefore, the increased desoption energy $(1.9 \mathrm{eV})$ in this work prevents the hydrogen atoms from leaving the system at lower temperatures, thereby encouraging surface diffusion till the atom gets trapped or recombined. If they recombine, they are released as hydrogen molecules else they are released as atoms at the temperatures corresponding to the desorption energy (with migration energy $1.9 \mathrm{eV}$ ). 
It was also observed that as the inner surface area of the porous graphite sample increases, $\mathrm{T}_{50 \%}$ (the temperature at which both atomic and molecular hydrogen are released in equal amount) shifts towards lower temperatures and lesser hydrogen is released in molecular form at higher temperatures. The detailed description of the effect of inner surface area on retention and release of hydrogen from graphite will be published soon.

\section{Summary and Conclusions}

The two-region model proposed by Haasz et al. [1] was implemented to study the reactive diffusive transport of hydrogen in porous graphite. It was shown that the inner surface area of porous graphite available for transport of hydrogen plays a major role on hydrogen release [1]. However, several other factors influence the hydroegn release characteristics. Samples having similar inner surface areas but different void sizes and orientations show different hydrogen release characteristics. The effect of void fraction and void size on diffusion coefficients was studied in [7]. At temperatures below about $800 \mathrm{~K}$ most of the hydrogen atoms are trapped and negligible amount of hydrogen is released. For a hydrogen atom to be released from the material, must first diffuse along the inner surface (crystallite-microvoid interface) and then recombine with another hydrogen atom to form a hydrogen molecule. At higher temperatures $(\mathrm{T}>800 \mathrm{~K})$, trapped hydrogen atoms are released via de-trapping. The release of hydrogen from the graphite is rate-limited by diffusion, recombination and detrapping 
or a combination of all of these processes. Various factors affecting hydrogen retention and release were identified and their effects were studied. The proposed model was validated by comparing the model calculations with the experimental results for the retention and release of hydrogen in graphite. It was shown that at a given temperature, samples subjected to lower fluence have higher retention level. With increasing flux a larger number of hydrogen was released in molecular form even at higher temperatures and $\mathrm{T}_{50 \%}$ (the temperature at which both atomic and molecular hydrogen are released in equal amount) shifted towards higher temperatures. A similar effect was observed with an increase in the range of penetration of the ion beam. In both the cases the recombination effect was dominant over the other competiting factors at higher temperatures. The increase in the trap site density resulted in a higher number of the trapped hydrogen atoms but it did not seem to affect the overall retention and release characteristics. In the present code we predefine the number of hydrogen atoms in the sample and therefore we can not simulate the buildup of hydrogen inventory in the sample and then the re-emission after the steady state is achieved. As an improvement to the present code, we are planning to implement the true steady state with a continuous influx of hydrogen atoms decided by the flux of the ion beam in our simulations. This improvement in the code would allow us to understand the effect of the different particles arriving at different times in the simulation and the effect of this on their diffusion and recombination. We are also working on the coupling of TRIM to our code to get the self-consistent dynamical changes in the structure of the sample due to the incident energetic ion beam. By incorporating this, we can overcome the present limitation of our code where the number of trap sites are predefined. 


\section{Acknowledgements}

A. Rai and R. Schneider acknowledge funding of the work by the Initiative and Networking Fund of the Helmholtz Association. M. Warrier acknowledges funding from the Max-Planck India Fellowship. 


\section{References}

[1] A. A. Haasz, P. Franzen, J. W. Davis, S. Chiu, and C. S. Pitcher, J. Appl. Phys. 77 $(1995) 66$.

[2] W. Möller, J. Nucl. Mater. 162-164 (1989) 138.

[3] A. A. Haasz, S. Chiu, and J.W. Davis, Proceedings of the IAEA Technical Committee Meeting on Atomic and Molecular Data for Fusion Reactor Technology, Cadarache, France, October 12-16, 1992, edited by R.K. Janev and H.W. Drawin (IAEA, Cadarache, 1993), p. 92

[4] G. Federici and C.H. Wu, J. Nucl. Mater. 186 (1992) 131.

[5] J. Küppers, Surface Science Reports 22 (1995) 249.

[6] S. Chiu and A.A. Haasz, J. Nucl. Mater. 196-198 (1992) 972.

[7] M. Warrier, Multi-scale modeling of hydrogen isotope transport in porous graphite. Ph.D. Dissertation, Faculty of Mathematics and Natural Science, Uni. Greifswald, Germany, (2004).

[8] A. Hassanein, B. Wiechers, and I. Konkashbaev, J. Nucl. Mater. 258-263 (1998) 295.

[9] B. V. Mech, A. A. Haasz, and J. W. Davis, J. Appl. Phys. 84 (1998) 1655.

[10] B. M. U. Scherzer, M. Wielunski, W. Möller, A. Turos, and J. Roth, Nucl. Instrum. Methods Phys. Res. B 33 (1988) 714.

[11] B. M. U. Scherzer, J. Wang and W. Möller, J. Nucl. Mater. 162-164 (1989) 1013.

[12] W. Möller and B. M. U. Scherzer, J. Appl. Phys. 64 (1988) 4860. 
[13] R. A. Causey, J. Nucl. Mater. 162-164 (1989) 151.

[14] H. Atsumi, S. Tokura, and M. Miyake, Proc. 3rd Int. Conf. on Fusion Reactor Materials, Karlsruhe, (1987), J. Nucl. Mater. 155-157 (1988) 241.

[15] E. Salonen, K. Nordlund, J. Keinonen, and C. H. Wu, Phys. Rev. B 63 (2001) 195415.

[16] Y. Ferro, F. Marinelli, and A. Allouche, Chemical Physics Letters 368 (2003) 609.

[17] Y. Ferro, F. Marinelli, and A. Allouche, Journal of Chemical Physics 116 (2002) 8124.

[18] M. Warrier, R. Schneider, E. Salonen, and K. Nordlund, Physica Scripta T108 (2004) $85-89$.

[19] M. Warrier, R. Schneider, E. Salonen, and K. Nordlund, Contrib. Plasma Phys. 44 (2004) 307 .

[20] K. L. Wilson, R. Bastasz, R.A. Causey, D.K. Brice, B.L. Doyle, W.R. Wampler, W. Möller, B.M.U. Scherzer, T. Tanabe, Trapping, detrapping and release of implanted hydrogen isotopes, in: Atomic and plasma-material interaction data for fusion (supplement to the journal Nuclear Fusion) 1 (1991) 31.

[21] M. Warrier, R. Schneider, E. Salonen, and K. Nordlund, J. Nucl. Mater. 337-339 (2005) 580.

[22] M. V. Smoluchowski, Z. Physik Chem. 107 (1957) 463.

[23] T. R. Waite, Phy. Rev. 107 (1957) 463.

[24] G. Herzberg, Phys. Rev. Lett. 23 (1969) 1081.

[25] G. Duesing, H. Hemmerich, W. Sassin, and W. Schilling, Proceedings of the International 
Conference on Vacancies and Interstitials in Metals, Jülich, Germany, 1968, (NorthHolland, Amsterdam, 1970), p. 246

[26] P. Franzen and E. Vietzke, J. Vac. Sci. Technol. A 12 (1994) 820.

[27] J. W. Davis, A. A. Haasz and D.S. Walsh, J. Nucl. Mater. 176-177 (1990) 992-999.

[28] J.A. Sawicki, J. Roth and L.M. Howe, J. Nucl. Mater. 162-164 (1989) 1019-1024.

[29] M. Braun and B. Emmoth, J. Nucl. Mater. 128-129 (1984) 657.

[30] B.L. Doyle, W.R. Wampler and D.K. Brice, J. Nucl. Mater. 103 (1981) 513.

[31] B.M.U. Scherzer, R.A. Langley, W. Möller, J. Roth and R. Schultz, J. Nucl. Mater. 194 (1982) 497.

[32] R.A. Langley, R.S. Blewer and J. Roth, J. Nucl. Mater. 76-77 (1978) 313.

[33] R. Schneider, A. Rai, A. Mutzke, M. Warrier, E. Salonen and K. Nordlund in: 12th International Conference on Fusion Reactor Materials, 4-9 Dec. 2005, Santa Barbara, CA, USA. J. Nucl. Mater., in press. 


\section{$6 \quad$ Figure captions}

Fig. 1: Released amount of hydrogen atoms and molecules. Lines: model calculations from present work, points: data obtained from experiments, ref. [26]. Out of 2000 hydrogen atoms distributed initially in the sample around $10 \%$ hydrogen is relesed at $900 \mathrm{~K}$, increasing to $70 \%$ at $1500 \mathrm{~K}$ and finally saturating around $87 \%$ at $2100 \mathrm{~K}$.

Fig. 2: Amount of retained hydrogen as a function of temperature for different fluences. Data obtained in this work is compared with the retention data reported by Braun et al. [29] $\left(20 \mathrm{keV}, 8 \times 10^{16} D^{+}\right.$ions $\left./ \mathrm{cm}^{2}\right)$, Sawicki et al. [28] $(40 \mathrm{keV}$, $5 \times 10^{15} H$ Tions $\left./ \mathrm{cm}^{2}\right)$, Doyle et al. [30] $\left(1.5 \mathrm{keV}, 10^{16} D^{+}\right.$ions $\left./ \mathrm{cm}^{2}\right)$, Scherzer et al. [31] $\left(1.5 \mathrm{keV}, 5 \times 10^{17} \mathrm{D}^{+}\right.$ions $\left./ \mathrm{cm}^{2}\right)$, and Langley et al. [32] $\left(8 \mathrm{keV}, 10^{18}\right.$ Datoms $\left./ \mathrm{cm}^{2}\right)$.

Fig. 3: Released amount of hydrogen atoms and molecules as a function of temperature for different depths of penetration.

Fig. 4: Amount of retained hydrogen as a function of temperature for different depths of penetration.

Fig. 5: Released amount of hydrogen atoms and molecules as a function of temperature for different amount of initial hydrogen atoms distributed in the sample. The released amount of hydrogen at temperatures $(900 \mathrm{~K}, 1300 \mathrm{~K}, 1900 \mathrm{~K})$ and for the case with 2000 (case 1), 5000 (case 2) and 8000 (case 3) hydrogen atoms distributed initially in the sample are $(2 \%, 43 \%, 77 \%),(4 \%, 50 \%, 75 \%)$ and $(8 \%, 51 \%, 72 \%)$ respectively.

Fig. 6: Released amount of hydrogen atoms and molecules as a function of temperature 
for different trap site densities. The released amount of hydrogen for all the trap site densities considered here are similar, i.e. at temperatures $(900 \mathrm{~K}, 1300 \mathrm{~K}, 1900 \mathrm{~K})$ the released amount of hydrogen are $(2 \%, 43 \%, 77 \%)$. 


\section{$\begin{array}{ll}7 & \text { Figures }\end{array}$}

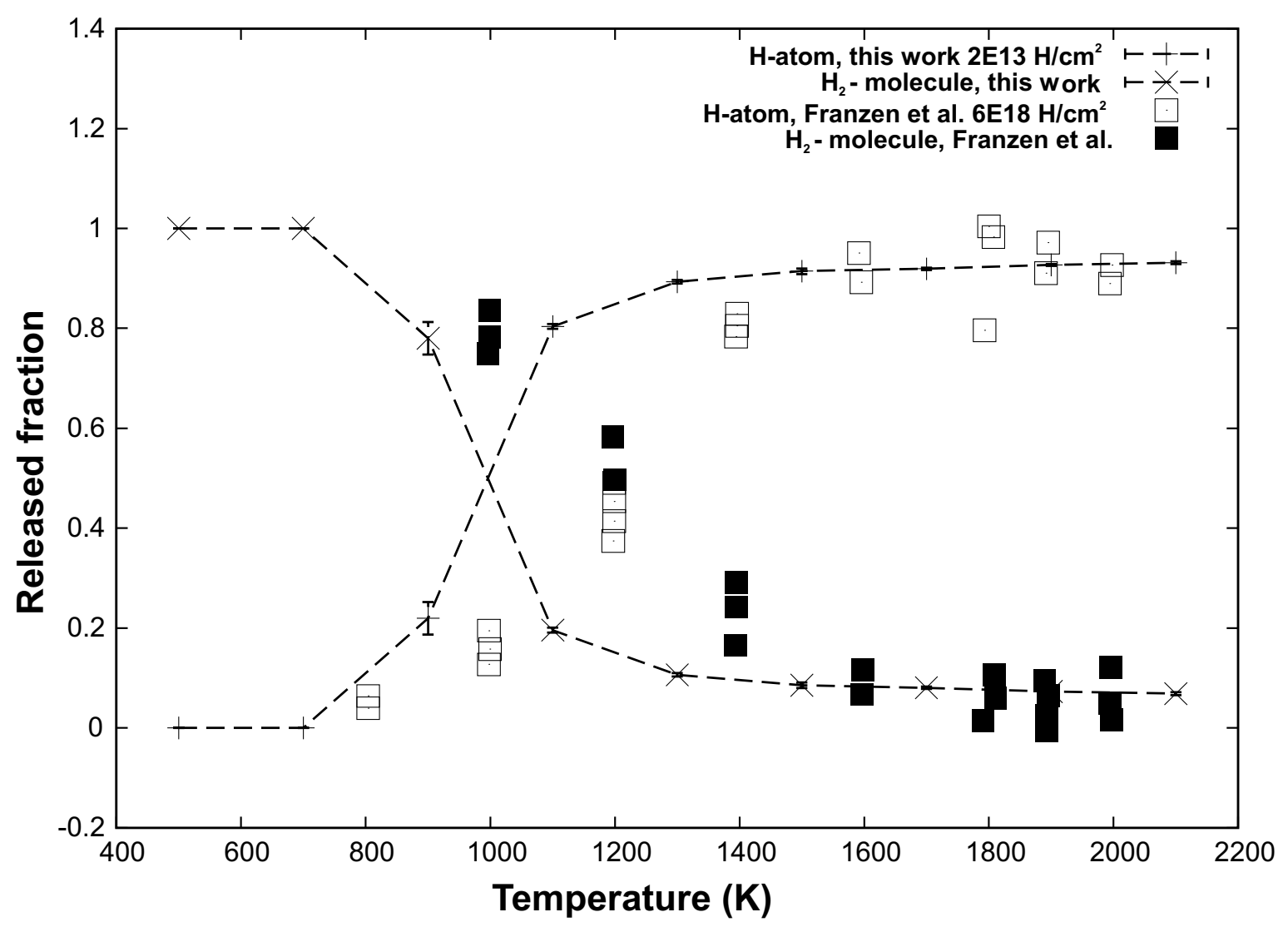

Fig. 1. 


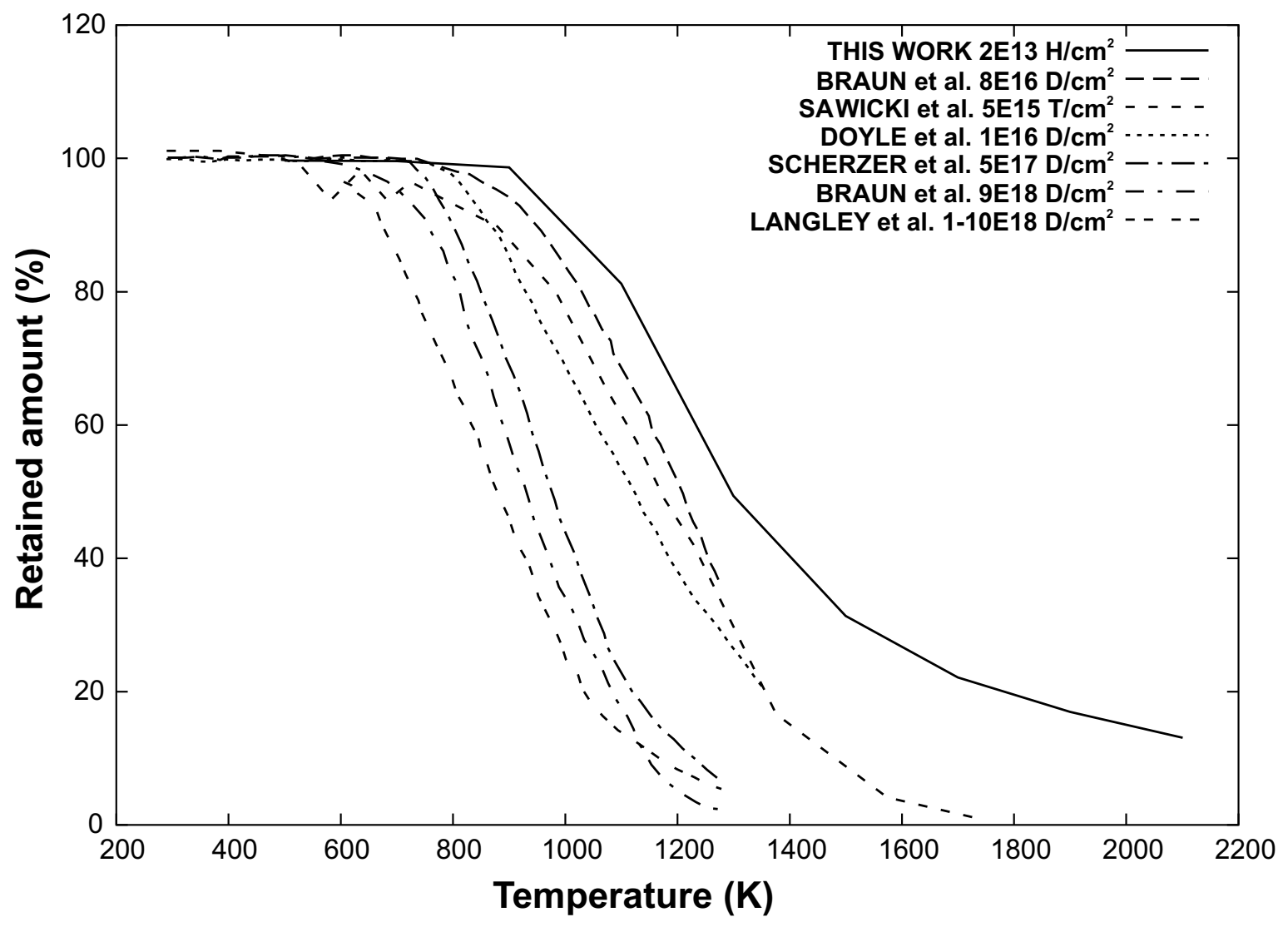

Fig. 2. 


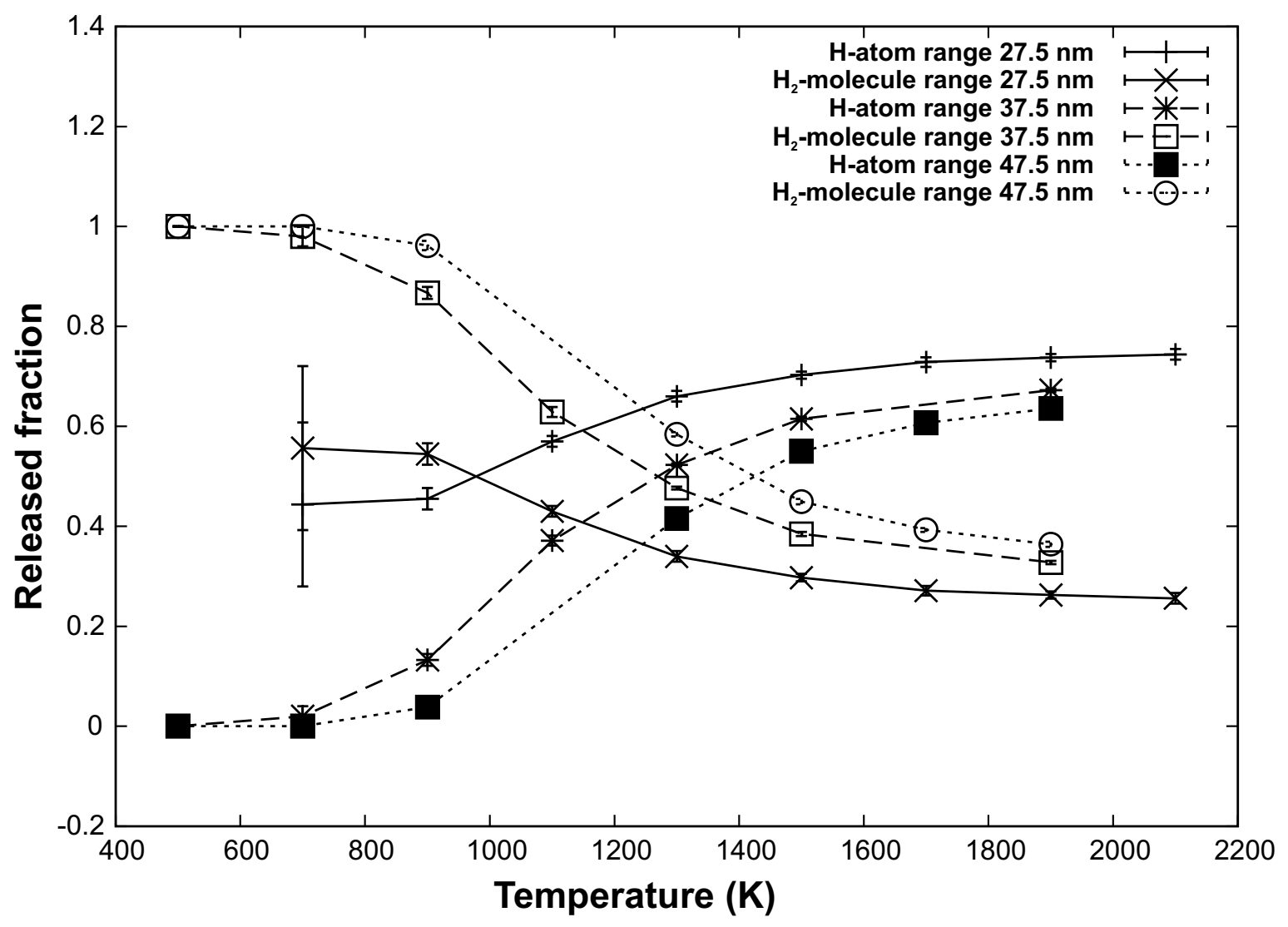

Fig. 3. 


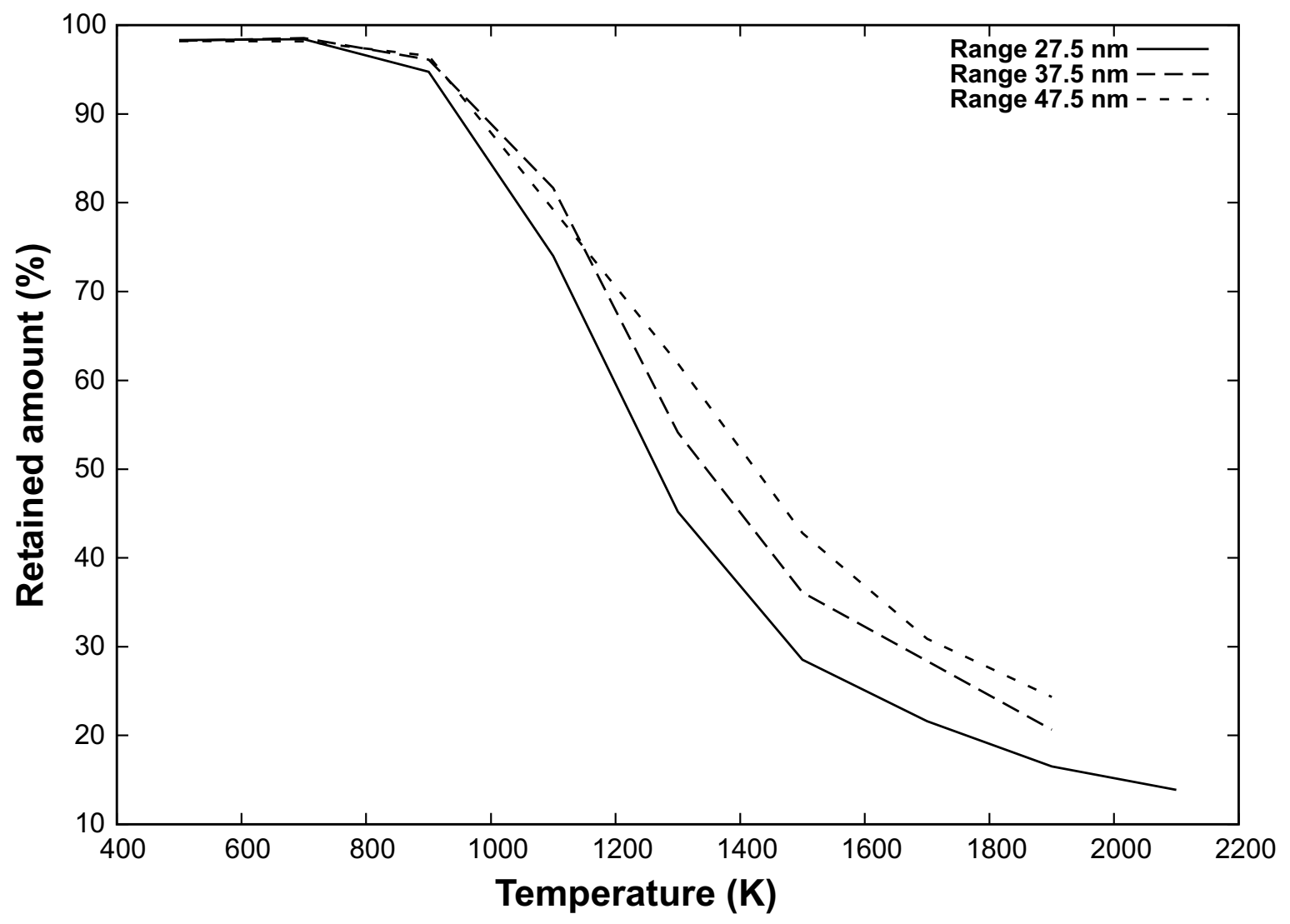

Fig. 4. 


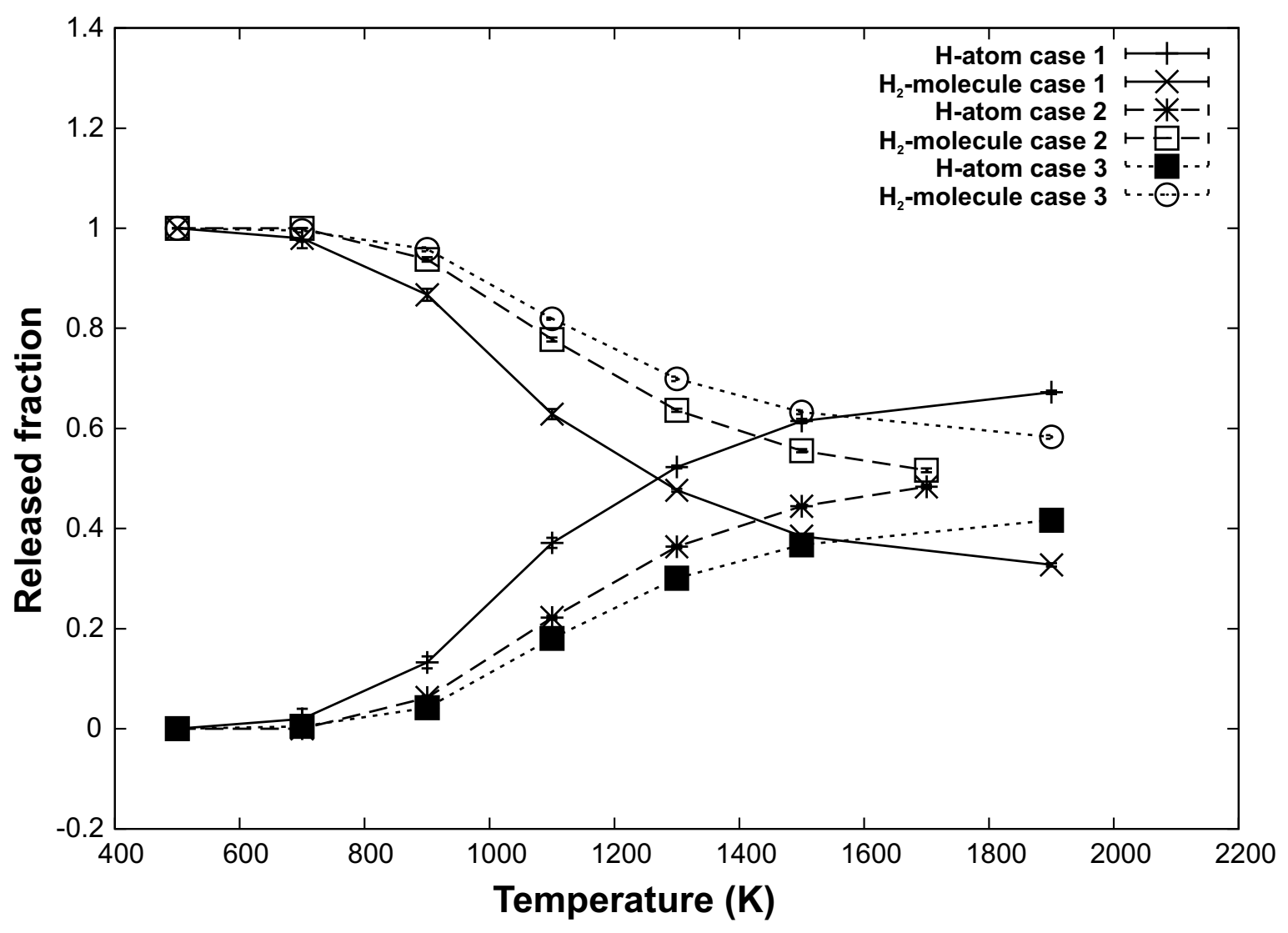

Fig. 5. 


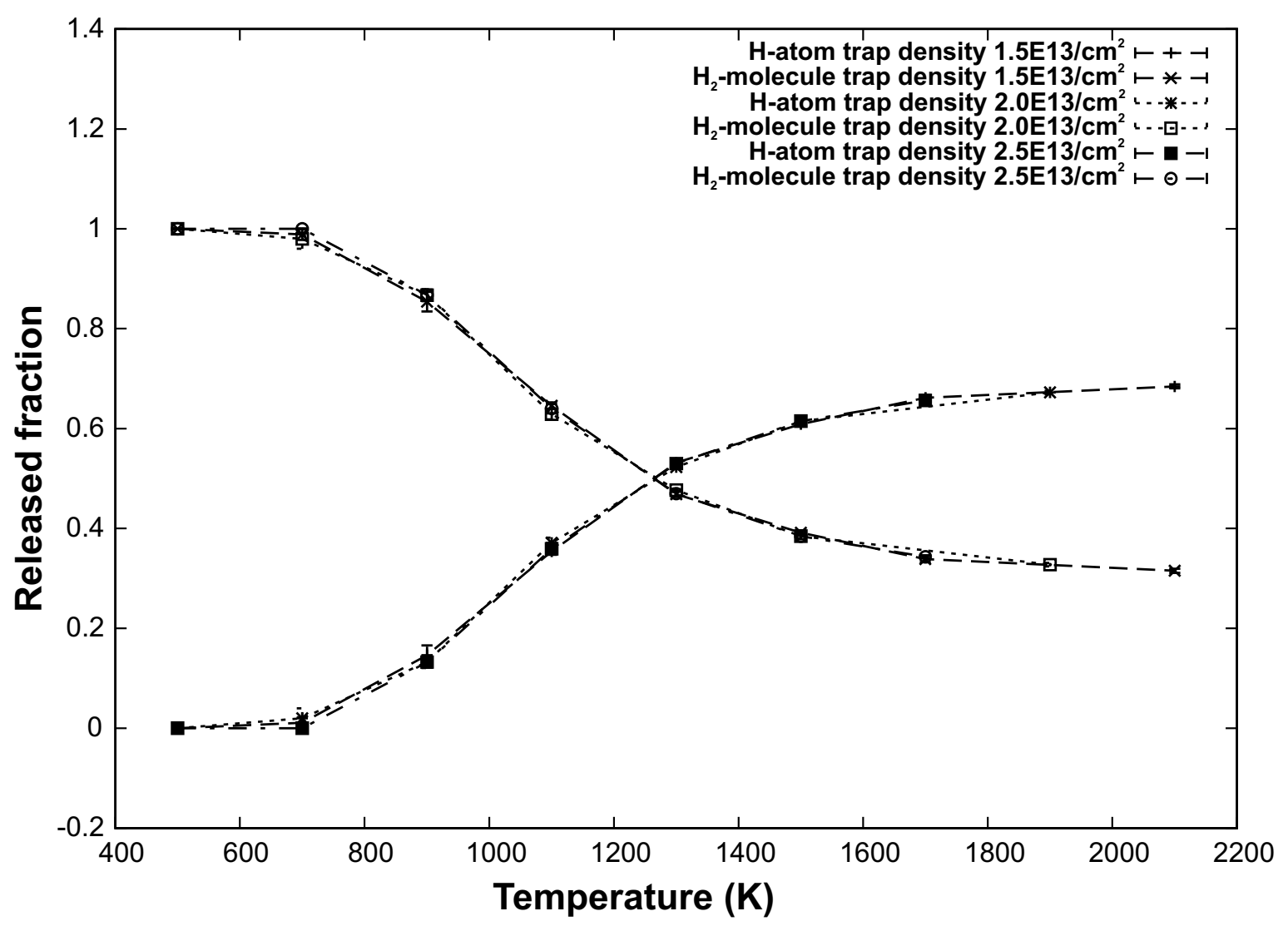

Fig. 6. 